\title{
Benchmarking a MOS-based Algorithm on the BBOB-2010 Noisy Function Testbed
}

\author{
Antonio LaTorre \\ Department of Computer \\ Systems Architecture and \\ Technology \\ Facultad de Informática \\ Universidad Politécnica de \\ Madrid, Spain \\ atorre@fi.upm.es
}

\author{
Santiago Muelas \\ Department of Computer \\ Systems Architecture and \\ Technology \\ Facultad de Informática \\ Universidad Politécnica de \\ Madrid, Spain \\ smuelas@fi.upm.es
}

\author{
José M. Peña \\ Department of Computer \\ Systems Architecture and \\ Technology \\ Facultad de Informática \\ Universidad Politécnica de \\ Madrid, Spain \\ jmpena@fi.upm.es
}

\begin{abstract}
In this paper, a hybrid algorithm based on the Multiple Offspring Sampling framework is presented and benchmarked on the BBOB-2010 noisy testbed. MOS allows the seamless combination of multiple metaheuristics in a hybrid algorithm capable of dynamically adjusting the participation of each of the composing algorithms. The experimental results show a good performance on functions with moderate noise. However, on functions with severe noise the results deteriorate, which suggests that further research should be conducted to find more adequate control mechanisms for these types of functions.
\end{abstract}

\section{Categories and Subject Descriptors}

G.1.6 [Numerical Analysis]: Optimization-global optimization, unconstrained optimization; F.2.1 [Analysis of Algorithms and Problem Complexity]: Numerical Algorithms and Problems

\section{General Terms}

Algorithms

\section{Keywords}

Benchmarking of algorithms, Black-box optimization, Continuous optimization, IPOP-CMA-ES, Differential Evolution, Multiple Offspring Sampling

\section{INTRODUCTION}

In this contribution, a hybrid algorithm constructed by means of the Multjple Offspring Sampling (MOS) framework [5] has been applied to the Black Box Optimization 2010 Noisy Function Testbed. This framework allows the combination of different evolutionary models following an $\mathrm{HRH}$
(High-level Relay Hybrid) approach (according to Talbj's taxonomy [8]) where the number of evaluations that each algorithm can carry out is dynamically adjusted according to their current performance. In this type of algorithms, two metaheuristics are executed in sequence, one after the other. For this paper, the IPOP-CMA-ES [1] and the DE algorithm [7] have been combined within this framework in a multistart strategy on 30 different functions. This algorithm is the same as the one presented in a complementary paper of the same proceedings [6].

\section{ALGORITHM AND PARAMETERS}

The algorithm and all parameters are described in the similar work on the Noiseless Testbed [6]. Due to the lack of time to do a proper parameter tuning on the noisy testbed, all the parameters values were kept the same as for the noiseless testbed.

\section{RESULTS}

Results from experiments according to [3] on the benchmark functions given in $[2,4]$ are presented in Figures 1, 2 and 3 and in Tables 1,2 and 3 .

The overall results in the noisy testbed are not as satisfactory as in the case of the noiseless one [6] in terms of achieved precision and scalability. The hybrid algorithm here presented is able to solve $30,27,25,19,16$ and 10 functions out of 30 in $2,3,5,10,20$ and 40 dimensions, respectively. It seems that the noise added to the functions makes the performance of the algorithm deteriorate as the number of dimensions increases. This effect is more pronounced in the case of those functions with severe noise than in those with a moderate noise.

Compared with the individual use of its composing algorithms, the DE seems not to be of much help in this type of functions. Fortunately, the regulatory mechanisms of the MOS framework are able to detect this behavior and minimize the participation of the DE technique. As a consequence of this, the overall behavior of the hybrid algorithm is quite similar to that exhibited by the CMA-ES when used individually, though it presents small variations for some groups of dimensions: in 2,3 and 40 dimensions it seems to have a better performance, whereas IPOP-CMA-ES seems to be slightly better in the rest of the dimensions $(5,10$ and 20). 

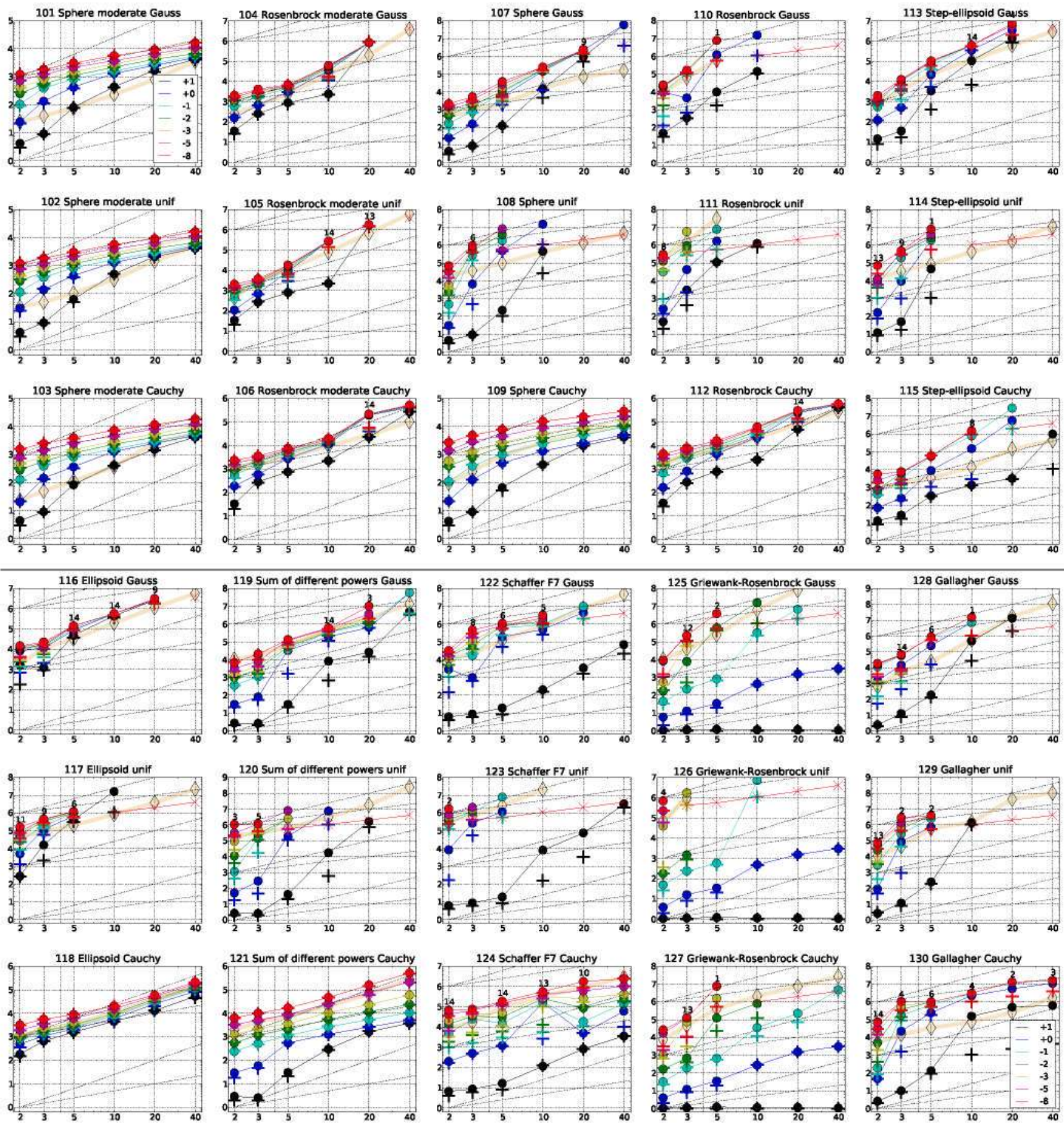

Figure 1: Expected Running Time (ERT, - ) to reach $f_{\text {opt }}+\Delta f$ and median number of $f$-evaluations from successful trials $(+)$, for $\Delta f=10^{\{+1,0,-1,-2,-3,-5,-8\}}$ (the exponent is given in the legend of $f_{101}$ and $f_{130}$ ) versus dimension in log-log presentation. For each function and dimension, ERT $(\Delta f)$ equals to \#FEs $(\Delta f)$ divided by the number of successful trials, where a trial is successful if $f_{\text {opt }}+\Delta f$ was surpassed. The \#FEs $(\Delta f)$ are the total number (sum) of $f$-evaluations while $f_{\text {opt }}+\Delta f$ was not surpassed in the trial, from all (successful and unsuccessful) trials, and $f_{\text {opt }}$ is the optimal function value. Crosses $(\times)$ indicate the total number of $f$-evaluations, \#FEs $(-\infty)$, divided by the number of trials. Numbers above ERT-symbols indicate the number of successful trials. Y-axis annotations are decimal logarithms. The thick light line with diamonds shows the single best results from BBOB-2009 for $\Delta f=10^{-8}$. Additional grid lines show linear and quadratic scaling. 

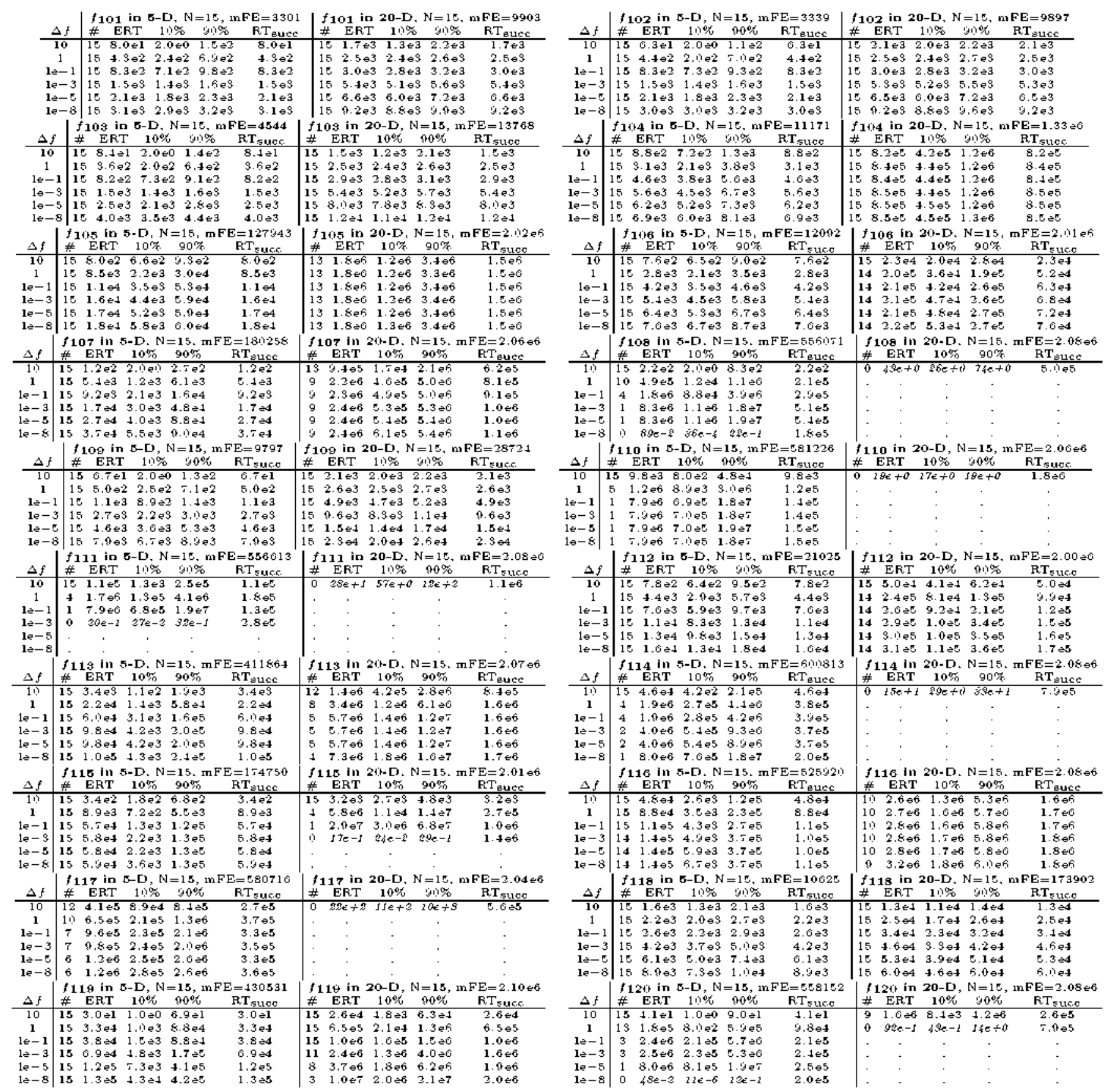

Table 1: Shown are, for functions $f_{101}-f_{120}$ and for a given target difference to the optimal function value $\Delta f$ : the number of successful trials (\#); the expected running time to surpass $f_{\mathrm{op} t}+\Delta f$ (ERT, see Figure 1); the $10 \%$-tile and $90 \%$-tile of the bootstrap distribution of ERT; the average number of function evaluations in successful trials or, if none was successful, as last entry the median number of function evaluations to reach the best function value $\left(\mathbf{R T}_{\text {succ }}\right)$. If $f_{\text {cpt }}+\Delta f$ was never reached, figures in italics denote the best achieved $\Delta f$-value of the median trial and the $10 \%$ and $90 \%$-tile trial. Furthermore, $\mathrm{N}$ denotes the number of trials, and $\mathbf{m F E}$ denotes the maximum of number of function evaluations executed in one trial. See Figure 1 for the names of functions. 

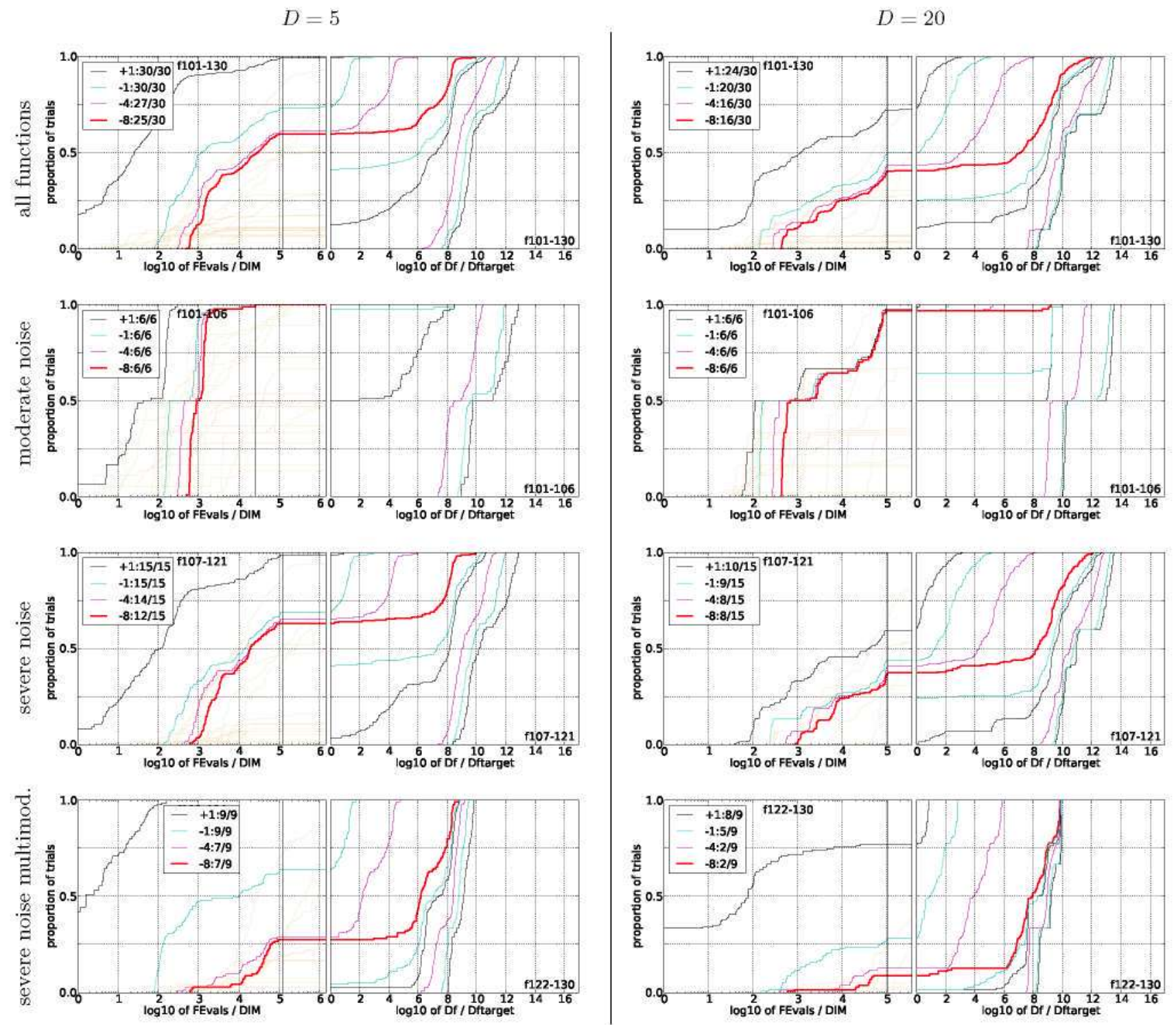

Figure 2: Empirical cumulative distribution functions (ECDFs), plotting the fraction of trials versus running time (left subplots) or versus $\Delta f$ (right subplots). The thick red line represents the best achieved results. Left subplots: ECDF of the running time (number of function evaluations), divided by search space dimension $D$, to fall below $f_{\text {opt }}+\Delta f$ with $\Delta f=10^{k}$, where $k$ is the first value in the legend. Right subplots: ECDF of the best achieved $\Delta f$ divided by $10^{k}$ (upper left lines in continuation of the left subplot), and best achieved $\Delta f$ divided by $10^{-8}$ for running times of $D, 10 \mathrm{D}, 100 \mathrm{D} \ldots$ function evaluations (from right to left cycling blackcyan-magenta). The legends indicate the number of functions that were solved in at least one trial. FEvals denotes number of function evaluations, $D$ and DIM denote search space dimension, and $\Delta f$ and Df denote the difference to the optimal function value. Light brown lines in the background show ECDFs for target value $10^{-8}$ of all algorithms benchmarked during BBOB-2009. 

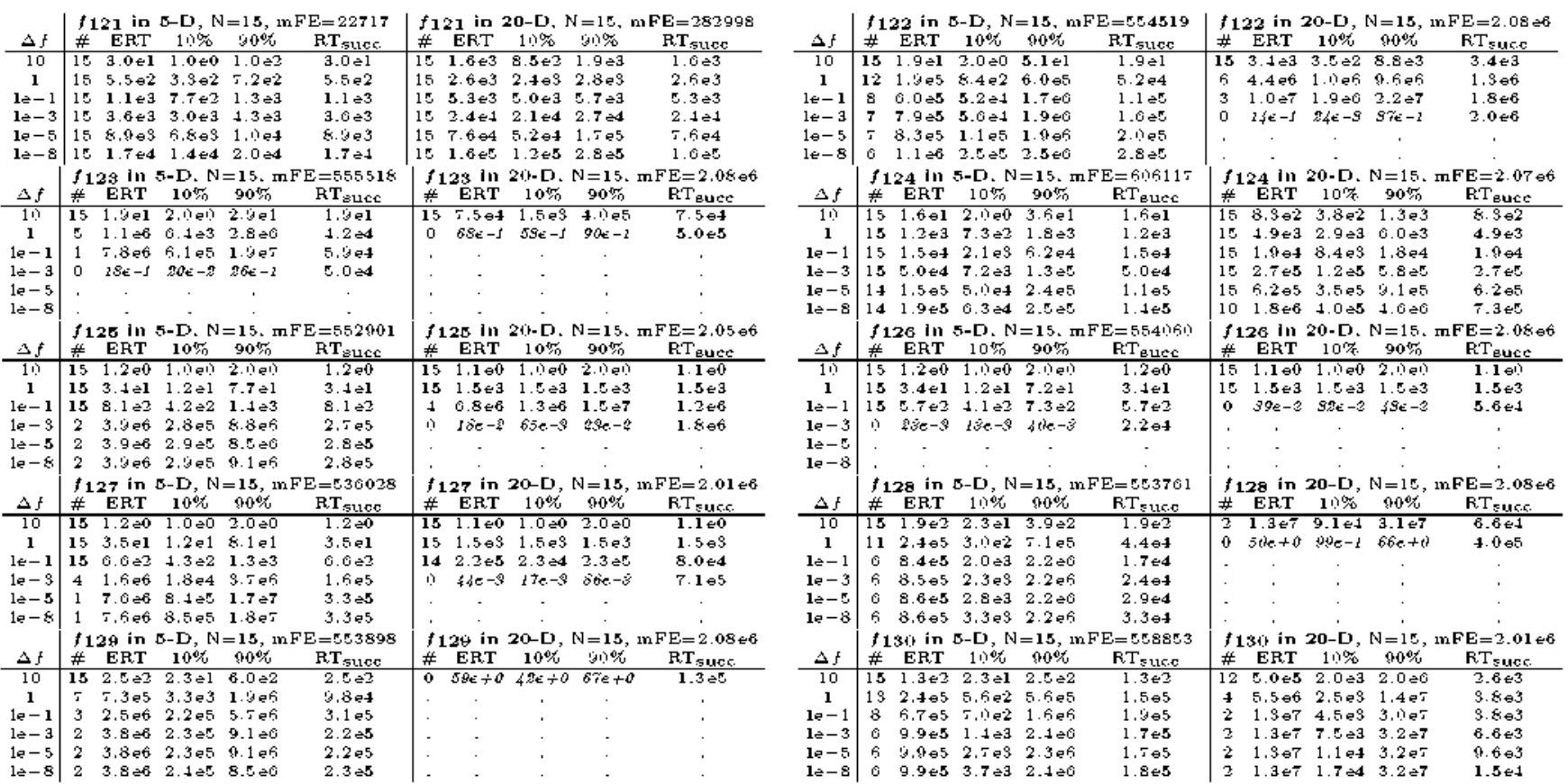

Table 2: Shown are, for functions $f_{121}-f_{130}$ and for a given target difference to the optimal function value $\Delta f$ : the number of successful trials (\#); the expected running time to surpass $f_{o \mathrm{pt}}+\Delta f$ (ERT, see Figure 1); the $10 \%$-tile and $90 \%$-tile of the bootstrap distribution of ERT; the average number of function evaluations in successful trials or, if none was successful, as last entry the median number of function evaluations to reach the best function value $\left(\mathbf{R T}_{\text {succ }}\right)$. If $f_{\text {opt }}+\Delta f$ was never reached, figures in italics denote the best achieved $\Delta f$-value of the median trial and the $10 \%$ and $90 \%$-tile trial. Furthermore, $\mathrm{N}$ denotes the number of trials, and $\mathbf{m F E}$ denotes the maximum of number of function evaluations executed in one trial. See Figure 1 for the names of functions.

Table 3: ERT loss ratio (see Figure 3) compared to the respective best result from BBOB-2009 for budgets given in the first column. The last row $R L_{U S} / D$ gives the number of function evaluations in unsuccessful runs divided by dimension. Shown are the smallest, $10 \%$-ile, $25 \%$-ile, $50 \%$-ile, $75 \%$-ile and $90 \%$ ile value (smaller values are better).

\begin{tabular}{|c|c|c|c|c|c|c|}
\hline & \multicolumn{6}{|c|}{$f_{101}-f_{130}$ in $5-\mathrm{D}, \operatorname{maxFE} / \mathrm{D}=1212$} \\
\hline $\mathrm{FEs} / \mathrm{D}$ & best & $10 \%$ & $25 \%$ & med & $75 \%$ & $90 \%$ \\
\hline 0 & 1.1 & 1.3 & 1.9 & & 5.6 & \\
\hline 10 & & & & & 6.6 & 29 \\
\hline 100 & 1. & 3.6 & 5.6 & 7.8 & 12 & \\
\hline $1 \mathrm{e}$ & & 2. & & - & & \\
\hline 1 & & & & & & \\
\hline & & 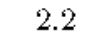 & & & & \\
\hline \multirow[t]{2}{*}{$\mathrm{RL}_{\mathrm{US}} / \mathrm{D}$} & $1 \mathrm{e} 5$ & $1 \mathrm{e} 5$ & $1 \mathrm{e} 5$ & $1 \mathrm{e} 5$ & $1 \mathrm{e} 5$ & $1 \mathrm{e} 5$ \\
\hline & \multicolumn{6}{|c|}{$f_{101}-f_{130}$ in $20-\mathrm{D}, \operatorname{maxFE} / \mathrm{D}=104808$} \\
\hline $\mathrm{Es} / \mathrm{D}$ & & $10 \%$ & $25 \%$ & med & $75 \%$ & $90 \%$ \\
\hline 2 & & 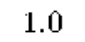 & 2.4 & & 40 & 40 \\
\hline 10 & & 3.0 & 18 & 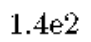 & $2.0 \mathrm{e}-2-3.0$ & 2.0 \\
\hline 100 & & 4.6 & 7.2 & & 84 & 2.0 \\
\hline & & 2.6 & 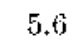 & & & 2.0 \\
\hline $1 \mathrm{e} 4$ & & 3.2 & 9.2 & (f) & $2.8 \mathrm{e} 2$ & 2.0 \\
\hline & & 2.6 & 4.4 & & 2 & $1.0 \mathrm{t}$ \\
\hline 16 & & 2.6 & 4.3 & 27 & 22 & 1. \\
\hline $\mathrm{Lus} / \mathrm{D}$ & $1 \mathrm{e} 5$ & $1 e 5$ & $1 \mathrm{e} 5$ & $1 \mathrm{e} 5$ & $1 \mathrm{e} 5$ & $1 \mathrm{e} 5$ \\
\hline
\end{tabular}

\section{CONCLUSIONS}

In this paper a hybrid algorithm combining Differential Evolution and IPOP-CMA-ES has been benchmarked on the BBOB-2010 noisy testbed. The experimental results show a good performance on functions with a moderate noise. On the other hand, functions with severe noise seem to be harder to solve with this algorithm. A more thorough study on the control mechanisms, specially those related to the detection of the stagnation and the restart of the search process, should be conducted on these functions. The selection of the parameter values was done based on the similar work of the noiseless testbed. Therefore, the proposed algorithm should also benefit of a proper parameter tuning process. Finally, the combination of additional techniques could be of great help in order to improve current results.

\section{ACKNOWLEDGMENTS}

This work was supported by the Madrid Regional Education Ministry and the European Social Fund and financed by the Spanish Ministry of Science TIN2007-67148. The authors thankfully acknowledge the computer resources, technical expertise and assistance provided by the Centro de Supercomputación y Visualización de Madrid (CeSViMa) and the Spanish Supercomputing Network. 

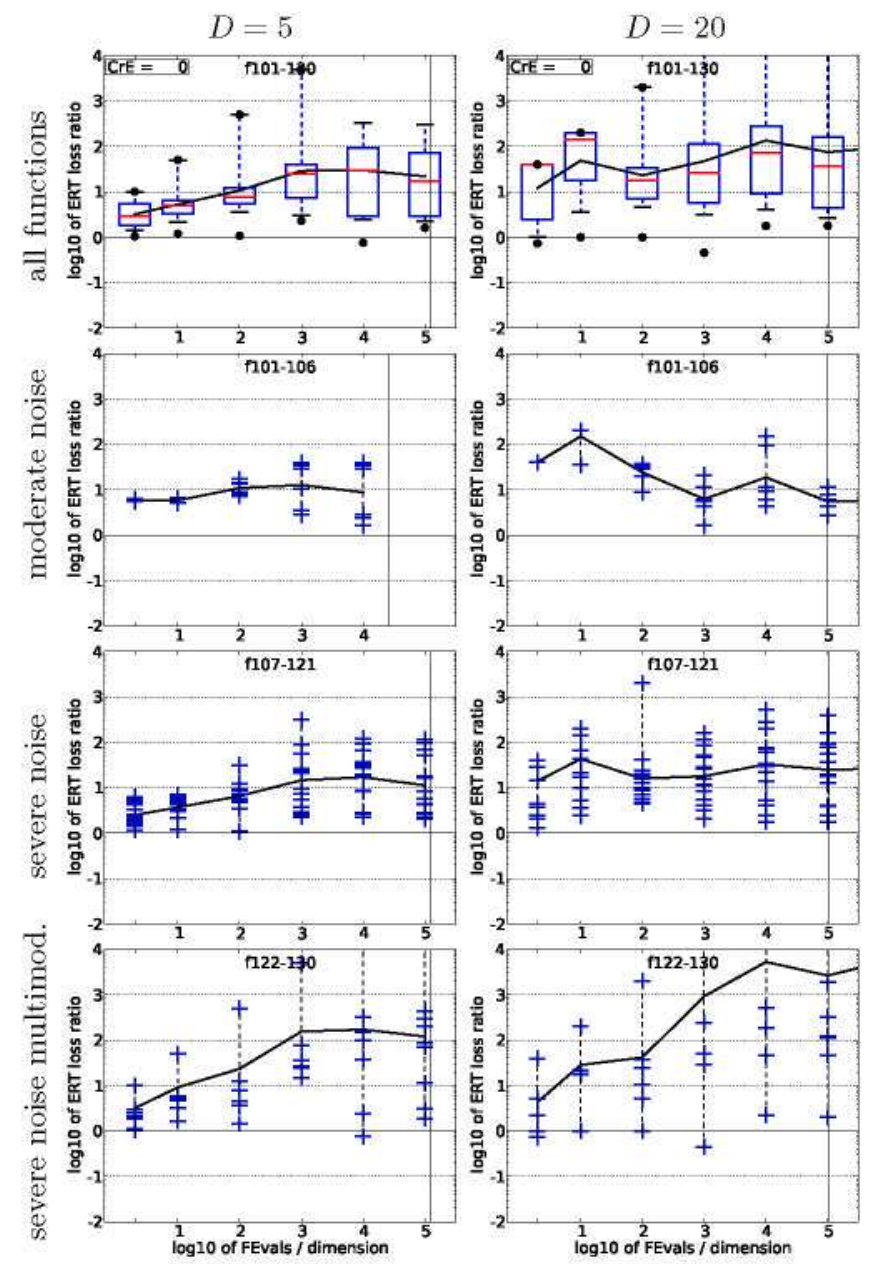

Figure 3: ERT loss ratio versus given budget FEvals. The target value $f_{\mathrm{t}}$ for ERT (see Figure 1 ) is the smallest (best) recorded function value such that ERT $\left(f_{\mathrm{t}}\right) \leq$ FEvals for the presented algorithm. Shown is FEvals divided by the respective best $\operatorname{ERT}\left(f_{\mathrm{t}}\right)$ from BBOB-2009 for functions $f_{101}-f_{130}$ in 5-D and 20D. Each ERT is multiplied by $\exp (\mathrm{CrE})$ correcting for the parameter crafting effort. Line: geometric mean. Box-Whisker error bar: 25-75\%-ile with median (box), 10-90\%-ile (caps), and minimum and maximum ERT loss ratio (points). The vertical line gives the maximal number of function evaluations in this function subset.

\section{REFERENCES}

[1] A. Auger and N. Hansen. A restart CMA evolution strategy with increasing population size. In Proceedings of the IEEE Congress on Evolutionary Computation, CEC 2005, pages 1769-1776. IEEE Press, 2005.

[2] S. Finck, N. Hansen, R. Ros, and A. Auger. Real-parameter Black-Box Optimization Benchmarking 2010: Presentation of the noisy functions. Technical Report 2009/21, Research Center PPE, 2010.

[3] N. Hansen, A. Auger, S. Finck, and R. Ros. Real-parameter Black-Box Optimization Benchmarking 2010: Experimental setup. Technical Report RR-7215, INRIA, 2010.

[4] N. Hansen, S. Finck, R. Ros, and A. Auger. Real-parameter Black-Box Optimization Benchmarking 2009: Noisy functions definitions. Technical Report RR-6869, INRIA, 2009. Updated February 2010.

[5] A. LaTorre. A Framework for Hybrid Dynamic Evolutionary Algorithms: Multiple Offspring Sampling (MOS). PhD thesis, Universidad Politécnica de Madrid, November 2009.

[6] A. LaTorre, S. Muelas, and J. Peña. Benchmarking a MOS-based algorithm on the BBOB-2010 noiseless function testbed. In Workshop Proceedings of the GECCO Genetic and Evolutionary Computation Conference. ACM, 2010.

[7] R. Storn and K. Price. Differential evolution - A simple and efficient adaptive scheme for global optimization over continuous spaces. Technical report, 1995.

[8] E.-G. Talbi. A taxonomy of hybrid metaheuristics. Journal of Heuristics, 8(5):541-564, September 2002. 